\title{
Depressive rumination is correlated with brain responses during self-related processing
}

\author{
Tzu-Yu Hsu, PhD*; Tzu-Ling Liu, PhD; Paul Z. Cheng, MA; Hsin-Chien Lee, MD; \\ Timothy J. Lane, PhD; Niall W. Duncan, PhD*
}

\begin{abstract}
Background: Rumination, a tendency to focus on negative self-related thoughts, is a central symptom of depression. Studying the self-related aspect of such symptoms is challenging because of the need to distinguish self effects from the emotional content of task stimuli. This study employed an emotionally neutral self-related paradigm to investigate possible altered self-processing in depression and its link to rumination. Methods: People with major depressive disorder $(n=25)$ and controls $(n=25)$ underwent task-based electroencephalogram recording. We studied late event-related potentials, along with low-frequency oscillatory power. We compared electroencephalogram metrics between groups and correlated them with depressive symptoms and reported rumination. Results: Participants with major depressive disorder displayed a difference in late positive potentials across frontocentral electrodes between self-related and non-self-related conditions. We found no such difference in controls. The magnitude of this difference was positively correlated with depressive symptoms and reported rumination. Participants with major depressive disorder also had elevated theta oscillation power at central electrodes in self-related conditions, a finding that we did not see in controls. Limitations: Patients with major depressive disorder were medicated at the time of the study. The group studied was primarily female, so the observed effects may have been sex-specific. Conclusion: Rumination appears to be linked to altered self-related processing in depression, independent of stimuli-related emotional confounds. This connection between self-related processing and depression may point to a self disorder as a core component of depression.
\end{abstract}

\section{Introduction}

Rumination involves repetitive thoughts about feelings and problems. ${ }^{1}$ It is a common symptom in major depressive disorder (MDD), in which the thoughts tend to focus on negative emotions and life problems. This focus distinguishes depressive rumination from constructive repetitive thought, which can be of benefit to an individual. ${ }^{2}$ The tendency for negative rumination in MDD is correlated with the severity and duration of depressive episodes (see Nolen-Hoeksema and colleagues ${ }^{3}$ for a review), as well as with suicide risk ${ }^{4}$ and the likelihood of relapse in those recovering from MDD. 5,6 Rumination also appears to be a risk factor for developing MDD in healthy people ${ }^{7}$ and to predict its onset. ${ }^{8}$ Investigating the root neural changes leading to maladaptive rumination is an important step toward understanding MDD and individual differences in vulnerability.

A fundamental feature of rumination is that the thoughts involved are self-related. Based on this, a relationship has been proposed between the mechanisms of rumination and the mechanisms of self-related thought. ${ }^{9}$ Such a supposition is supported by an overlap in the brain regions involved in processing each. More specifically, self-related thought has been linked to brain regions along the cortical midline, such as the medial prefrontal and posterior cingulate cortices. ${ }^{10}$ Activity properties in these same regions have been further linked to individual differences in general rumination and are modulated by a rumination task. ${ }^{11-13}$ These cortical midline structures have also been linked to depressive symptoms and negative self-focused thought. ${ }^{14,15}$ Finally, activity in cortical midline structures during self-referential thinking appears to differ between patients with MDD and controls. ${ }^{16-18}$ Together, this evidence suggests that investigating changes to self-related processing may provide important insights into ruminative symptoms in MDD.

Investigating self-related processing in MDD presents a methodological challenge, because many of the tasks used involve stimuli that have emotional valence as well as being self-related. For example, a commonly used task asks participants to relate particular positive or negative personal traits

Correspondence to: N. W. Duncan and T. J. Lane, Graduate Institute of Mind, Brain and Consciousness, Taipei Medical University, 250 Wuxing Street, Taipei, Taiwan; niall.w.duncan@gmail.com; timlane13@gmail.com

*These authors contributed equally to this work.

Submitted Apr. 6, 2021; Revised May 20, 2021; Accepted Jun. 11, 2021

Cite as: J Psychiatry Neurosci 2021 September 21;46(5). doi: 10.1503/jpn.210052 
to themselves or to others. ${ }^{17,18}$ This task induces a late positive potential (LPP) - a component linked to motivationally salient stimuli ${ }^{19}$ — over frontocentral regions that differ between patients with depression and controls. ${ }^{20-22}$ However, the emotional valence of the stimuli used in such tasks may interact with the negative emotional bias found in depression. ${ }^{23}$ This means that people with depression tend to focus more on negative self-related stimuli and interpret stimuli more negatively in relation to themselves. ${ }^{24-26}$ With this potential interaction between the self-related stimuli used and an inherent negative bias, it becomes challenging to parse the contribution to brain activity measures of self-related processing in itself from the influence of altered emotional processing.

Tasks that use emotionally neutral stimuli may circumvent this issue and allow more direct study of self-related processing in MDD. One such task was developed by Johnson and colleagues, ${ }^{27}$ in which sets of 3 coloured patches are used as the stimulus. In one task condition, participants are asked to judge which set of patches they prefer; in the other, they are asked which set is most similar in terms of their relative positions in colour space. These 2 conditions respectively represent self-related (subjective: "which do I prefer") and nonself-related (objective: "which is most like the other") judgments. An increase in central-frontal activity is observed in the self-related condition, in line with the described relationship between cortical midline structures and self-related processing. ${ }^{27-29}$ These brain responses are likely to be independent of task-induced emotional biases and provide a tool for investigating the relationship between self-related processing and rumination without also probing negative bias.

The present study took advantage of this colour judgment task, in conjunction with electroencephalogram (EEG) recordings, to investigate the association between self-related processes and rumination in a group of patients with MDD, along with age- and sex-matched healthy controls. We assessed ruminative behaviours using the Ruminative Responses Scale (RRS), ${ }^{30}$ and we used an ERP approach to analyze the EEG data, focusing on the LPPs identified in previous work. We predicted that self and non-self ERPs would differ between patients and controls at central-frontal electrodes, and that these differences would be correlated with RRS scores. Because LPPs have been linked to theta oscillations, ${ }^{19,31}$ which have in turn been connected to selfrelated processing, ${ }^{32}$ we also investigated changes in this frequency band during the task. Our approach advanced previous work linking self-related processes and rumination into the temporal domain in a manner that may have been less biased by differences in emotional processing.

\section{Methods}

\section{Participants}

We recruited 25 patients diagnosed with MDD (20 female; age $38.6 \pm 13.5$ years [mean \pm standard deviation]) at the Shuang-Ho Hospital Department of Psychiatry. Diagnosis was made according to the DSM-IV. We used a MINI structured interview ${ }^{33}$ to confirm diagnosis, detect suicide risk and exclude patients with comorbid mental or substance use disorders. Patients with poor visual acuity or a history of neurologic issues were also excluded. The average duration of the most recent depressive episode was $4.1 \pm 3.15$ months. All patients but 1 were medicated at the time of testing (see Appendix 1, Table S1, available at jpn.ca). Patients had been on the same medication schedule for at least 2 weeks before participation and were not receiving any nonpharmacological treatment. Twenty-five control participants (21 females; age $38.2 \pm 14.2$ years) with no history of neurologic or psychiatric disorders, and who were not using any psychotropic medication, were recruited from the community. Groups were age- and sex-matched, apart from 1 pair, in which the sexes differed. The study was approved by the Taipei Medical University Institutional Review Board (N201603080). Written consent was obtained from all participants before participation. Control participants were compensated financially for their time.

\section{Questionnaires}

We assessed ruminative behaviours in both patients and controls using the 22-item RRS. ${ }^{30} \mathrm{We}$ administered the 21-item Beck Depression Inventory (BDI) ${ }^{34}$ to both patients and controls to evaluate the presence of general depressive symptoms across both groups. Both questionnaires were presented in Traditional Chinese. ${ }^{35,36}$

\section{Colour judgment task}

The general configuration of the task stimuli is illustrated in Figure 1. Each trial began with a 1500-2000 ms fixation cross (subtending $0.5^{\circ} \times 0.5^{\circ}$ ). A stimulus array consisting of 3 nonoverlapping, differently coloured squares was then presented for $2000 \mathrm{~ms}$. One square was located at the middle top, and the remaining 2 were located at the left and right bottom. In each trial, the top square was the target to which the other 2 were compared. Each coloured square subtended a visual angle of $1.5^{\circ} \times 1.5^{\circ}$.

We selected 3 colours for each trial from a set of 12 taken from the CIELAB colour space at $30^{\circ}$ intervals around the $a^{*}$ (red-green) $-b^{*}($ yellow-blue $)$ plane colour wheel. Trial difficulty was determined by the objective similarity in hue between the target and the 2 lower squares. In low-difficulty trials, the 2 choice colours would be $60^{\circ}$ and $150^{\circ}$ from the target colour. In high-difficulty trials, the relative difference in distance would be less $\left(90^{\circ}\right.$ and $\left.120^{\circ}\right)$, making it more difficult to discriminate the degree of similarity to the target. The luminance of the 3 squares was equalized by manipulating the red, green and blue components of the colours.

The task consisted of 2 different conditions. During the colour similarity judgment condition, participants were required to judge which of the lower squares was most similar in hue to the target and then press the corresponding left or right mouse button (Figure 1). For the preference judgment condition, participants were instructed to choose the colour pairing they preferred between the target and either of the lower squares. We used the same low- and high-difficulty 


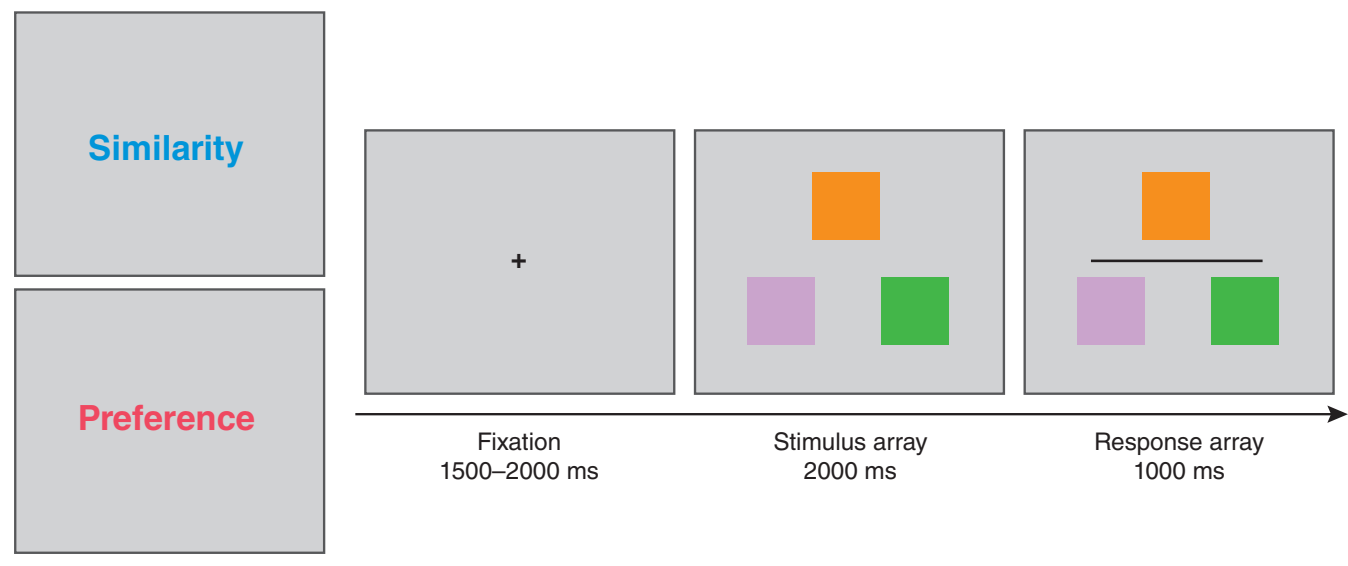

Figure 1: At the start of each block, participants were instructed to make a judgment about similarity or preference. Each trial started with a fixation period of 1500-2000 ms, followed by the stimulus and response arrays. The stimulus array consisted of 3 colour swatches on which participants made their judgment. Then, a dark grey horizontal line appeared to indicate the start of the response period, during which participants would press the left or right button to indicate their decision.

sets of colours in the preference trials as in the similarity trials. After a viewing period, participants had to respond within $1000 \mathrm{~ms}$. This response period was indicated by a dark grey horizontal line. We used a relatively long decisionmaking period and a separate response period to reduce any differences in response timing that may have arisen from psychomotor symptoms in MDD. ${ }^{37}$

Participants completed 20 practice trials before performing 4 blocks of 48 trials. The preference judgment and similarity judgment conditions were organized in a block design. Participants were told which condition they would perform before each block. Each block was initiated by the participant, allowing for breaks between blocks to reduce fatigue. Participants were instructed to maintain visual fixation on the centre of the screen during each trial while avoiding blinks and eye movements. The sequence of conditions was counterbalanced across participants.

The experiment was programmed in Psychtoolbox $3^{38}$ running on MATLAB R2015a (MathWorks). Stimuli were displayed on a 24-inch liquid crystal display monitor with a vertical refresh rate of $60 \mathrm{~Hz}$. Participants viewed the stimuli at a distance of $57 \mathrm{~cm}$, and a chin rest was used to stabilize their head position.

\section{EEG recording and preprocessing}

We recorded EEGs with $\mathrm{Ag} / \mathrm{AgCl}$ electrodes mounted in an elastic cap (Easycap; Brain Products $\mathrm{GmbH}$ ) using a 30-electrode arrangement following the international 1020 system, including monopolar electrodes (FP1/2, $\mathrm{F} 7 / 3 / \mathrm{Z} / 4 / 8, \mathrm{FC} 5 / 3 / \mathrm{Z} / 4 / 6, \mathrm{~T} 7 / 8, \mathrm{C} 3 / \mathrm{Z} / 4, \mathrm{CP} 5 / 1 / \mathrm{Z} / 2 / 6$, $\mathrm{P} 7 / 3 / \mathrm{Z} / 4 / 8, \mathrm{O} 1 / \mathrm{Z} / 2)$. We attached an additional 6 channels to measure eye movement and to use as references. We recorded vertical eye movement with 2 electrodes on the supraorbital and infraorbital ridges of the left eye; we recorded horizontal eye movement with 2 electrodes on the outer canthi of the right and left eyes. The remaining 2 electrodes, attached to mastoid sites, served as references (A1 and $\mathrm{A} 2$ ). We kept impedances below $10 \mathrm{k} \Omega$ for all electrodes. We recorded online EEG using BrainAmp with a bandpass filter of $0.05-1000 \mathrm{~Hz}$. We recorded data using BrainVision Recorder 1.2, with a sampling rate of $1000 \mathrm{~Hz}$.

For offline analysis, we conducted EEG preprocessing using the EEGLAB ${ }^{39}$ (RRID:SCR_007292) and ERPLAB ${ }^{40}$ (RRID:SCR_009574) toolboxes in MATLAB. Continuous data were first low-pass filtered at a cut-off frequency of $30 \mathrm{~Hz}$. After manually excluding noisy periods, we conducted independent component analysis on the continuous waveforms to identify signals caused by eye movement, blinks and changes in muscle tone. We removed the contribution of these task-irrelevant signals using independent component analysis in EEGLAB. We then segmented continuous data into $2200 \mathrm{~ms}$ epochs, including $200 \mathrm{~ms}$ prestimulus and $2000 \mathrm{~ms}$ poststimulus intervals. Epochs were baselinecorrected with the prestimulus interval. Finally, epochs that contained signals exceeding $\pm 60 \mathrm{mV}$ in any channel were rejected, and the remaining trials averaged into the following categories: "similarity-low," "similarity-high," "preferencelow" and "preference-high."

\section{Behavioural data analysis}

We compared performance during similarity trials in terms of reaction times and accuracy using a 2 (difficulty) $\times$ 
2 (group) mixed analysis of variance (ANOVA). Preference trials had no objective criteria, so their accuracy could not be analyzed. As such, we compared reaction times for preference trials between groups through an independent-sample $t$ test. Finally, any interaction between condition and group for reaction times was tested using a 2 (condition) $\times 2$ (group) mixed ANOVA. Behavioural data were not available for 1 control participant.

\section{Analysis of task-induced response differences}

We analyzed event-related potentials (ERPs) induced by the task to identify any overall differences in brain responses between patients with MDD and controls. We tested the time period from 900 to $2000 \mathrm{~ms}$, covering sustained self-related components. ${ }^{20,21,41}$ Responses were averaged within this period at 12 electrodes along the midline and in the left and right hemispheres at the frontal $(\mathrm{Fz} / \mathrm{F} 3 / 4)$, central $(\mathrm{Cz} / \mathrm{C} 3 / \mathrm{C} 4)$, parietal (Pz/P3/P4) and occipital (Oz/O1/O2) scalp. We compared these using a mixed ANOVA that included a between-subject factor of group (control, MDD) and withinsubject factors of condition (similarity, preference), difficulty (low, high) and anterior-posterior location (frontal, central, parietal, occipital). We used post hoc tests to identify which anterior-posterior locations showed a difference between conditions in each group. We corrected $p$ values for sphericity using Greenhouse-Geisser adjustment. We applied Holm-Bonferroni adjustment to control the family-wise error rate. We conducted statistical analyses using JASP ${ }^{42}$ (RRID:SCR_015823).

We further analyzed groups of electrodes where we observed group $\times$ condition interactions to establish more precisely the times and locations at which differences between conditions occurred. We compared waveforms from individual electrodes within the selected electrode groups at each time point between conditions using paired $t$ tests. We used threshold-free cluster-based thresholding to identify periods of time across multiple electrodes in which conditions differed. ${ }^{43}$

\section{Self versus non-self response differences and depressive rumination}

Having established the specific time periods and electrodes at which activity differed between conditions, we then investigated the relationship between activity in these periods and depressive rumination. We used the time points at specific electrodes in which we saw a difference between conditions in the MDD group as a mask to extract average activity from the MDD and control groups. We then correlated these averaged activity levels with BDI scores separately for each group
(Spearman correlation). We also tested differences in correlation strength using linear regression. We then conducted a similar analysis using RRS scores.

\section{Self-related versus non-self-related processes and midline oscillatory power}

In a final step, we calculated induced response power at the frontocentral midline electrodes with LPP differences (Fz and $\mathrm{Cz}$ ), focusing on the alpha and theta bands. We selected these frequency ranges given their suggested connections to selfrelated processing. ${ }^{32}$ We calculated power spectral density for the period from stimulus onset to $2000 \mathrm{~ms}$. We separated oscillatory power from the $1 / f$ aperiodic component of the signal using the Fooof toolbox ${ }^{44}$ running on Python 3.7.9. Theta power was averaged from 4 to $8 \mathrm{~Hz}$ and alpha power from 8 to $13 \mathrm{~Hz}$. We then used mixed-effect linear regression to examine an oscillatory power fixed-effect group $\times$ condition interaction (with subject as a random effect). We used Holm-Bonferroni adjustment to correct $p$ values for the 2 electrodes and 2 frequency bands analyzed.

\section{Results}

\section{Questionnaire results}

Patients with MDD had significantly higher BDI and RRS scores than controls (Table 1).

\section{Behavioural results}

In the similarity task, participants were more accurate in the low-difficulty condition than in the high $\left(86.5 \%\right.$ v. $60.5 \% ; F_{1,47}=$ $186.04, p<0.001)$. Overall accuracy did not differ between groups $\left(F_{1,47}=2.54, p=0.12\right)$, nor was there a group $\times$ difficulty interaction $\left(F_{1,47}=0.023, p=0.87\right)$. Reaction times in the similarity task were faster in the low-difficulty condition than in the high (636 ms v. $\left.655 \mathrm{~ms} ; F_{1,47}=16.1, p<0.001\right)$. We found no effect of group on reaction time $\left(F_{1,47}=0.37, p=0.55\right)$, and no group $\times$ difficulty interaction $\left(F_{1,47}=0.038, p=0.84\right)$. Reaction times in the preference task did not differ between groups $\left(t_{47}=\right.$ $0.73, p=0.46)$. We found no interaction between task and group for reaction times $\left(F_{1,47}=0.037, p=0.84\right)$. Response accuracy and reaction times are given in Table 2 .

\section{Task-induced response differences}

Task-induced ERP amplitudes during the period from 900 to $2000 \mathrm{~ms}$ are summarized in Figure 2. We observed no difficulty $\times$ condition $\times$ group $\times$ location interaction $\left(F_{1.51,72.25}=\right.$

Table 1: Beck Depression Inventory and Rumination Response Scale

\begin{tabular}{lccc}
\hline Scale $^{*}$ & Control & MDD & Comparison \\
\hline Beck Depression Inventory score & $7.8 \pm 7.7$ & $28.7 \pm 14.4$ & $t=-6.7, p<0.001$ \\
Rumination Response Scale score & $40.4 \pm 12.5$ & $62.8 \pm 14$ & $t=-6.1, p<0.001$ \\
\hline *Values are mean \pm standard deviation. & & & \\
\hline
\end{tabular}


Table 2: Behavioural responses in each task condition for control and MDD groups

\begin{tabular}{lccc}
\hline & \multicolumn{2}{c}{ Similarity } & \\
\cline { 2 - 3 } Response* $^{*}$ Low & High & Preference \\
\hline Accuracy & & & \\
Control & $0.89 \pm 0.07$ & $0.64 \pm 0.11$ & - \\
MDD & $0.84 \pm 0.15$ & $0.59 \pm 0.16$ & - \\
Reaction time, ms & & & $637 \pm 102$ \\
Control & $627 \pm 107$ & $647 \pm 100$ & $655 \pm 99$ \\
MDD & $646 \pm 104$ & $664 \pm 97$ & \\
\hline
\end{tabular}

MDD = major depressive disorder

*Values are mean \pm standard deviation.

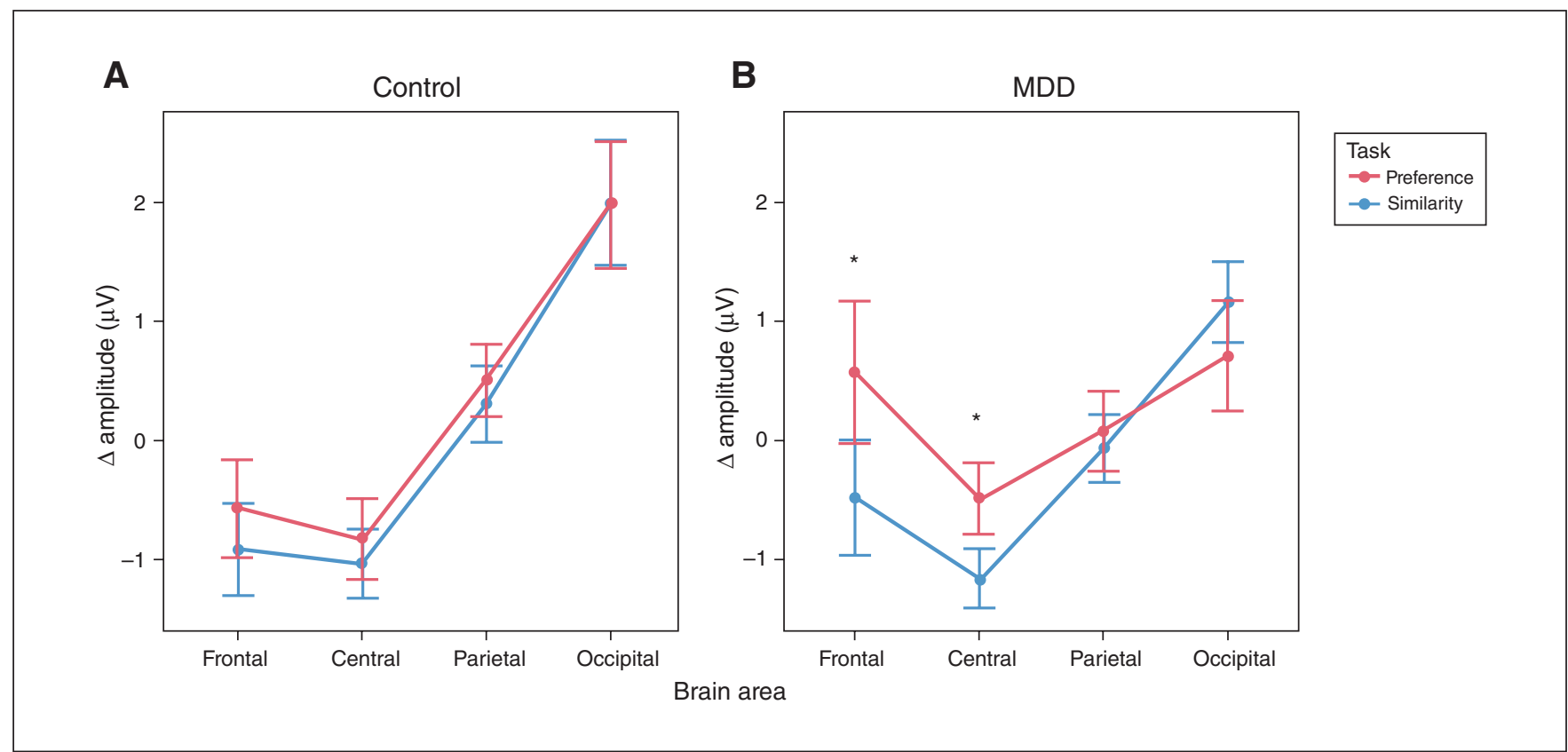

Figure 2: Mean event-related potential amplitudes at different scalp areas, 900-2000 ms after onset of the stimulus array, shown separately for (A) the control group and (B) the MDD group. Responses during the similarity condition are shown in blue, and responses during the preference condition are shown in red. We observed differences between the conditions at frontal and central areas in the MDD group but not in the control group. Error bars represent the standard error of the mean. ${ }^{*} p<0.05$. MDD $=$ major depressive disorder.

$0.76, p=0.44)$. As a result, we collapsed the difficulty factor for all subsequent analyses. We then observed an interaction between condition, group and location $\left(F_{1.5,71.77}=4.20, p=\right.$ $0.03)$. Follow-up simple main-effect analyses highlighted differences at the frontal and central locations. More specifically, the MDD group displayed a more negative waveform during the similarity condition (compared with the preference condition) at the frontal $(p<0.001)$ and central $(p=0.001)$ areas, but not the parietal $(p=0.44)$ or occipital $(p=0.06)$ areas. In contrast, the control group showed no amplitude differences between these conditions at any location (frontal $p=0.12$; central $p=0.17$; parietal $p=0.11$; occipital $p=0.89$ ).

Investigating the frontal and central locations in more detail, we conducted comparisons between conditions at each time point and at each relevant electrode. We observed significant differences between preference and similarity waveforms for the MDD group, starting at the frontal electrodes around $815 \mathrm{~ms}$ and then propagating to the central area (Figure 3A). During this period, measured activity became less negative over time for the preference condition than for the similarity condition. We observed no differences between conditions for the control group (Figure 3B).

\section{Self versus non-self response differences and depressive rumination}

As shown in Figure 4A, the differences in amplitude between similarity and preference conditions were positively correlated with BDI scores in the MDD group $\left(r_{24}=0.61, p=0.001\right)$, but not in the control group $\left(r_{24}=-0.36, p=0.08\right.$; Figure 4$)$. The correlations differed from each other $\left(t_{46}=3.01, p=0.004\right)$. We found a similar correlation between RRS scores and 
A
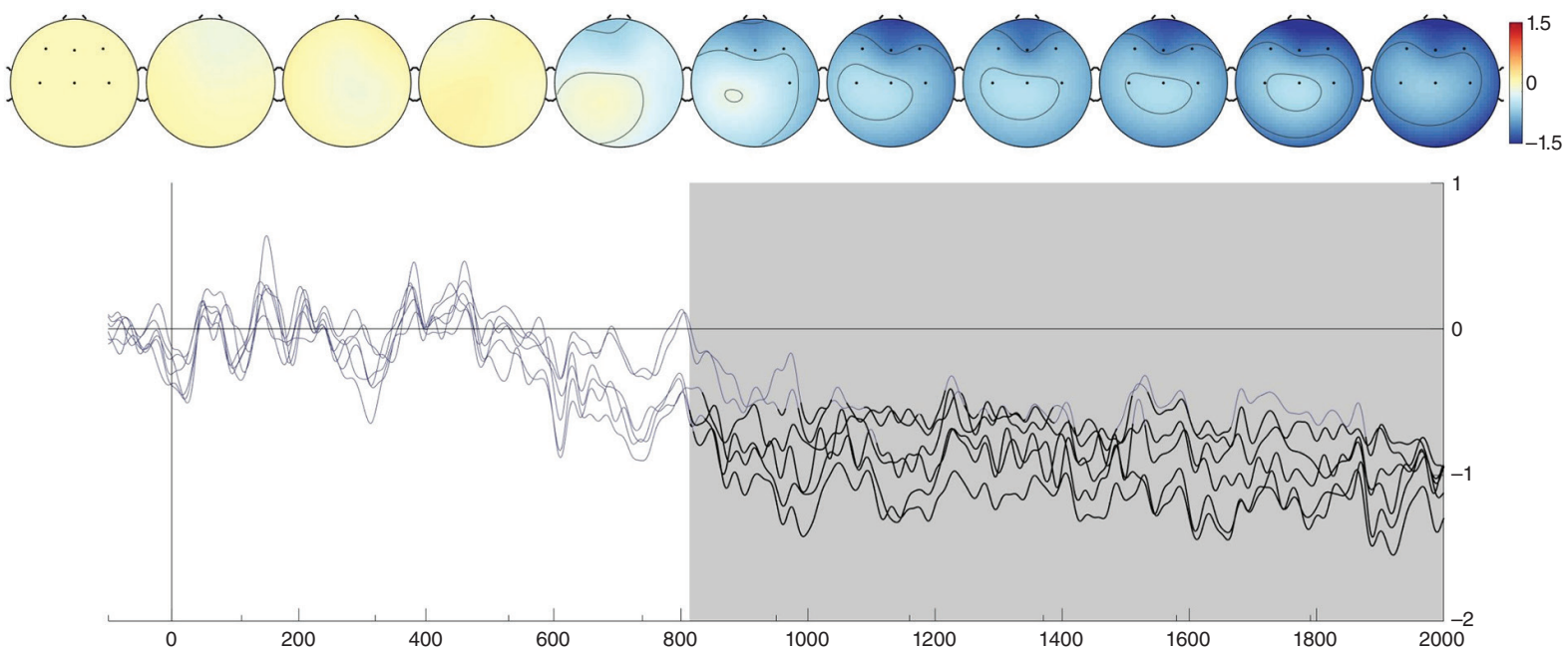

B
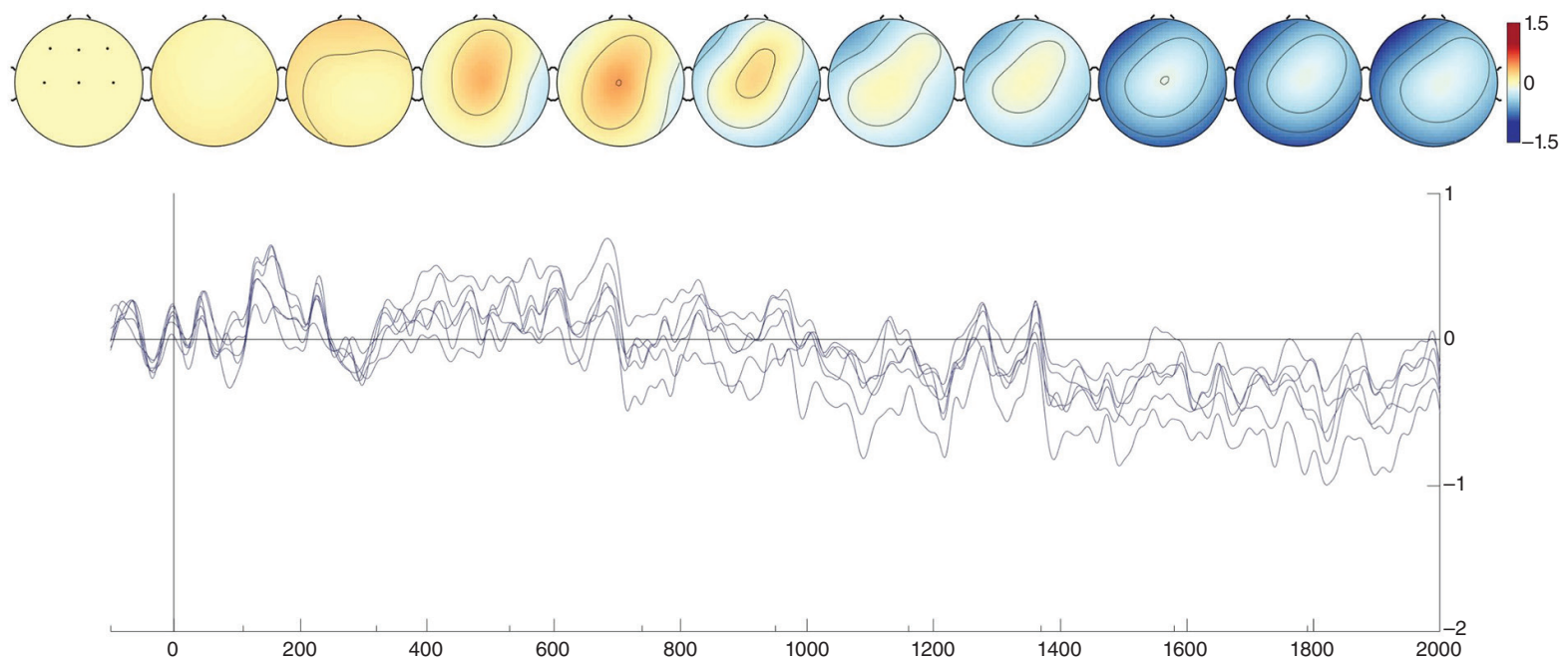

Figure 3: Similarity-preference differences for (A) the MDD group and (B) the control group. The electrodes for which comparisons were done are indicated by black dots in the leftmost topographical maps. These maps show similarity-preference differences over time. The waveforms represent similarity-preference differences for each selected electrode. Significant differences per electrode are shown in black. Periods in which at least 1 difference was found are shaded in grey. Visualizations were created using the EEGVIS toolbox. ${ }^{45} \mathrm{MDD}=$ major depressive disorder.

amplitude differences in the MDD group $\left(r_{24}=0.48, p=0.016\right)$ but not controls $\left(r_{24}=-0.13, p=0.54\right.$; Figure $\left.4 \mathrm{~B}\right)$. These correlations also differed from each other $\left(t_{46}=2.43, p=0.019\right)$.

Self-related versus non-self-related processes and midline oscillatory power

Analyzing the interaction between task condition and group, we observed no effects at the Fz electrode for oscillatory power in either the theta $(\beta=0.033, z=0.4, p>0.99$; Appendix 1, Figure S1) or alpha bands $(\beta=-0.023, z=-0.61, p>$ 0.99; Appendix 1, Figure S2). In contrast, we did observe an interaction between condition and group in the theta band at the $\mathrm{Cz}$ electrode ( $\beta=-0.12, z=2.92, p=0.016$; Figure 5$)$. We found no such effect for alpha power at this electrode ( $\beta=$ $-0.002, z=-0.054, p>0.99$; Appendix 1, Figure S3). Post hoc $t$ tests (Holm-Bonferroni adjusted) showed that patients with MDD had higher theta power in the preference condition than in the similarity condition $\left(t_{24}=-2.65, p=0.038\right)$. Theta power did not differ between conditions for control participants $\left(t_{24}=1.08, p=0.6\right)$.

\section{Discussion}

We investigated the relationship between self-related activity and depressive rumination using an emotionally neutral task 
A

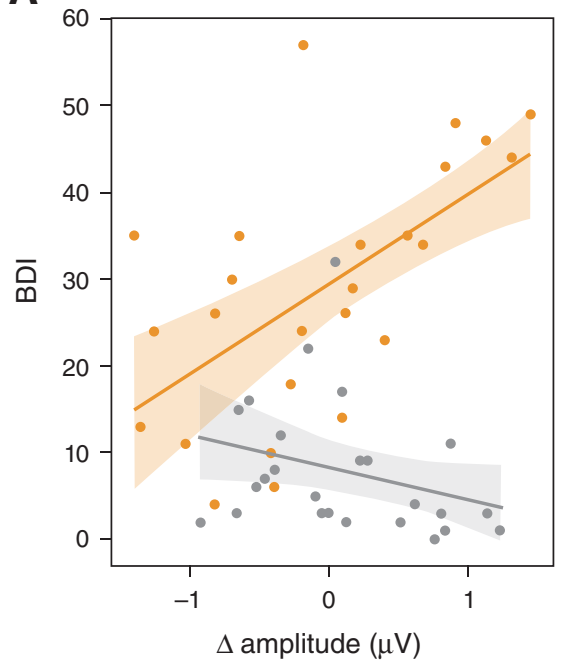

B

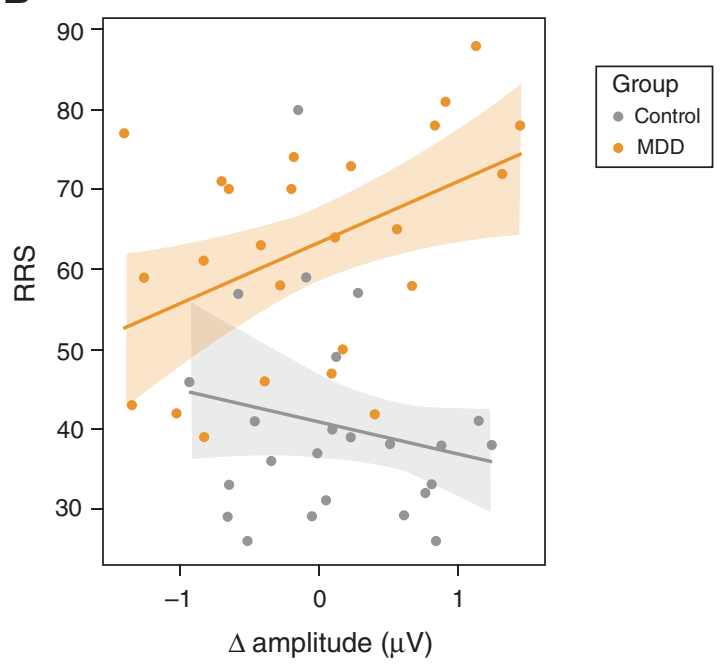

Figure 4: Differences in the amplitude of sustained responses between preference and similarity conditions were correlated with scores on (A) the $\mathrm{BDI}$ and $(\mathrm{B})$ the RRS (MDD group, orange; control group, grey). We observed significant relationships between amplitude differences and scores for participants with MDD but not for controls. BDI = Beck Depression Inventory; MDD = major depressive disorder; RRS = Rumination Response Scale.
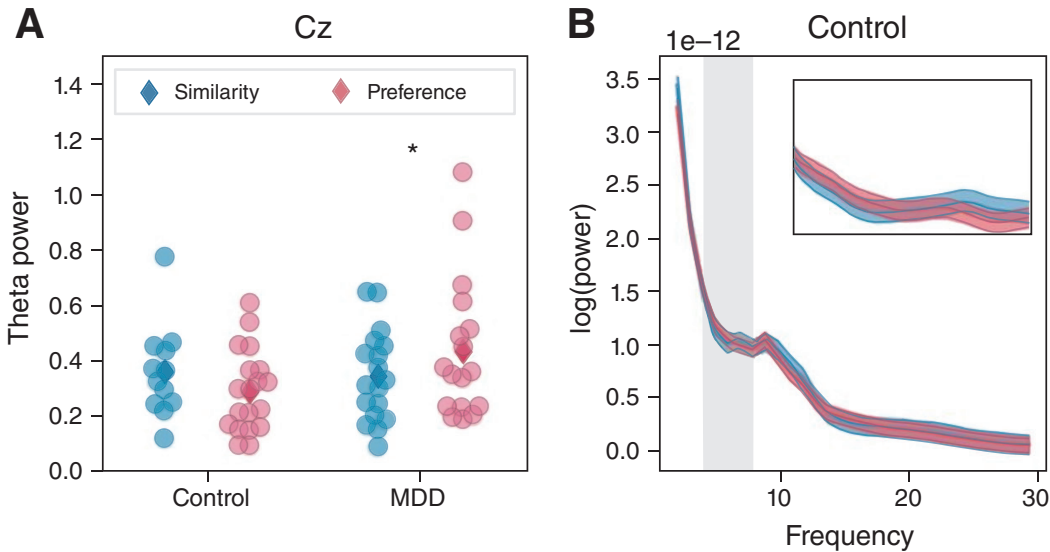

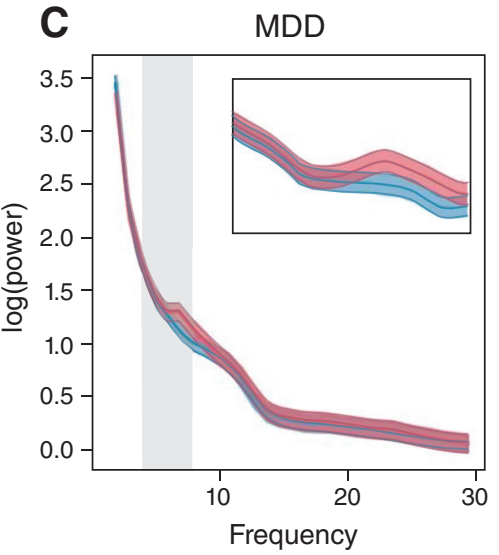

Figure 5: (A) Theta band oscillatory power at $\mathrm{Cz}$ as a function of condition for the MDD and control groups. Dots represent values from individuals in the similarity (blue) and preference (red) conditions. Group means are indicated by diamonds. Power spectra from which we obtained values are shown for $(B)$ the control group and $(C)$ the MDD group. Insets show a close-up of the theta range indicated by grey shading in the main spectra. ${ }^{*} p<0.05$. MDD $=$ major depressive disorder.

in patients with MDD and healthy controls. We observed differences between groups in a late ERP component at the frontal and central electrodes. Patients with MDD displayed less negative activity than controls during the subjective preference condition than during the objective similarity condition. This amplitude difference between the similarity and preference conditions was positively correlated with BDI and RRS scores in the MDD group. Moreover, we found that an increase in central theta power occurred during the preference condition in participants with MDD.

In terms of behavioural performance, we observed no difference between groups in accuracy or reaction times for the similarity or preference conditions. Reaction times were faster in low-difficulty trials than in high-difficulty ones, 
suggesting that participants engaged with the task appropriately. This finding was equally true for patients with MDD and controls, as demonstrated by the lack of interaction between difficulty and group. The lack of differences in reaction times between patients with MDD and controls contrasted with other tasks, in which people with MDD tended to respond more slowly. ${ }^{46}$ Our finding can likely be explained by the experimental design, which gave participants a relatively long decision-making period while the target array was displayed $(2000 \mathrm{~ms})$ and gave no instructions to respond as quickly as possible. Taken together, these behavioural measures show that the colour similarity and preference task were completed equally well by both patient and control groups.

We observed sustained late ERPs at frontocentral electrodes in the present study. Previous ERP studies of selfrelated processing have identified similar LPPs. Those experiments employed a self-reference task in which participants attributed positive or negative words to themselves. ${ }^{47}$ In such tasks, patients with depression tended to link negative words to themselves and controls selected more positive ones. ${ }^{17}$ At the level of LPPs, patients displayed responses of greater amplitude over frontocentral regions for negative words than for positive words. This was in contrast to controls, in whom positive words induced greater evoked responses. ${ }^{20-22}$ In patients, the magnitude of the difference between LPPs induced by positive and negative words appeared to increase with symptom severity, ${ }^{48}$ suggesting that people with depression may selectively elaborate on negative emotional self-related information. Notably, and in contrast to these previous studies, the task used here did not involve an emotional component. The presence of these sustained late ERPs in the context of the task used in the present study suggests that LPP-like effects in general may be driven at least in part by self-related processes that are independent of emotional content.

In this study, we found a sustained difference in LPP-like activity between self-related and non-self-related stimuli until trial end in patients with MDD but not controls. Notably, the difference in this activity between conditions was positively correlated with reported ruminative behaviours in the MDD group. The LPP-like reactivity of patients with MDD in an emotionally neutral context may mean that people with depression are inclined toward engaging in continual thought whose nature is fundamentally selfrelated. This would be distinct from thought that is primarily emotional in nature and that therefore also has a selfrelated aspect as a secondary feature. This is in line with previous work linking rumination in depression to activity in regions such as the medial prefrontal cortex and posterior cingulate cortex ${ }^{49,50}$ which are activated by self-related processes independent of emotion. ${ }^{51}$ A connection between activity in the medial prefrontal cortex and posterior cingulate cortex and this LPP-like activity is further supported by the fact that these regions were more active during the preference condition in previous work that has employed the task in functional MRI. ${ }^{27}$ These associations may merit further research with tools that can better resolve temporal and spatial properties, such as magnetoencephalography or high-density EEG.

An analysis of oscillatory power at midline frontocentral electrodes revealed elevated $\mathrm{Cz}$ theta power during the preference condition, specifically in the MDD group. A range of previous work has found associations between theta band activity and self-related processing. ${ }^{32}$ This work also provides evidence for a connection between theta activity and cortical midline structures. The fact that only patients with MDD demonstrated an increase in theta power during the self-related preference condition suggests that people with depression may be more sensitive to the subjectivity of experiences, generating a response that can be seen only with explicit self-cueing in people without depression. This sensitivity may then lead to an increased likelihood to engage in self-related thought in a manner that leads to ruminative symptoms. However, such a suggestion remains speculative and requires further investigation. Finally, although we found no differences in alpha power at the frontocentral electrodes, previous work has drawn connections between hemispheric asymmetries in this band and both depressive symptoms ${ }^{52}$ and LPPs. ${ }^{53}$ Further work targeted at such asymmetries in the context of self-related processing and rumination may therefore be of interest.

\section{Limitations}

A number of limitations should be noted. The first of these is the medication status of participants: all but 1 were undergoing pharmacological treatment. A previous study found that selective serotonin reuptake inhibitors influenced selfreflective activity, but this highlighted subcortical regions and revealed no effect on cortical regions such as the medial prefrontal cortex or posterior cingulate cortex. ${ }^{54}$ Antidepressants may also influence EEG measures, but information relating to the ERPs and oscillations highlighted here is inconclusive. More work with unmedicated patients is required to exclude drug effects entirely. Finally, our participant group was primarily female. There is some evidence for sex differences in the relationship between self-related thought and depressive symptoms, in that there may be less of an association in men. ${ }^{55}$ The current results require replication in a balanced participant sample.

\section{Conclusion}

The current findings suggest that rumination is related to altered self-related processing in depression, and that this can be shown independent of potential confounds arising from task stimuli with explicit emotional content. The difference in neural responses induced by self-related stimuli in patients with MDD lends weight to a conception of MDD that includes self-disorder as a fundamental component. Future research may wish to focus on treatment options that engage more directly with the self, including both psychotherapeutic and pharmacological interventions.$^{56}$ Further work investigating similar processes in other conditions linked to modifications in self-processing or ruminative behaviours, such as psychosis or anxiety disorders, ${ }^{57,58}$ may also be enlightening. 
Acknowledgements: The authors thank all participants for their time and effort. They are grateful also to Hsin-Yi Wang and Ching Lin for help with patient recruitment and data collection.

Affiliations: From the Graduate Institute of Mind, Brain and Consciousness, Taipei Medical University, Taipei, Taiwan (Hsu, Liu, Cheng, Lane, Duncan); the Brain and Consciousness Research Centre, TMU Shuang-Ho Hospital, New Taipei City, Taiwan (Hsu, Cheng, Lane, Duncan); the Institute of Cognitive Neuroscience, National Central University, Zhongda, Taiwan (Liu); the Department of Psychiatry, School of Medicine, College of Medicine, Taipei Medical University, Taipei, Taiwan (Lee); and the Department of Psychiatry, Taipei Medical University Hospital, Taipei, Taiwan (Lee).

Data accessibility: Participants did not give consent to their data being shared openly; the data included in this analysis are available from the authors only upon reasonable request.

Funding: This work was supported by funding from the Taiwan Ministry of Science and Technology to T. Lane (105-2632-H038-001-MY3), T. Hsu (109-2410-H-038-010; 110-2410-H-038-009) and N. Duncan (108-2410-H-038-008-MY2). This work was also supported by the Taiwan Ministry of Education Higher Education Sprout Project (DP2-110-21121-01-N-10, DP2-110-21121-01-N-07-01) and by the Ministry of Health and Welfare (IIT-1091-9).

Competing interests: None declared.

Contributors: T. Hsu, T. Lane and N. Duncan designed the study. P. Cheng and H. Lee acquired the data, which T. Hsu, T. Liu and N. Duncan analyzed. T. Hsu, T. Liu and N. Duncan wrote the article, which P. Cheng, H. Lee and T. Lane reviewed. All authors approved the final version to be published and can certify that no other individuals not listed as authors have made substantial contributions to the paper.

Content licence: This is an Open Access article distributed in accordance with the terms of the Creative Commons Attribution (CC BY-NC-ND 4.0) licence, which permits use, distribution and reproduction in any medium, provided that the original publication is properly cited, the use is noncommercial (i.e., research or educational use), and no modifications or adaptations are made. See: https:/ / creativecommons.org/licenses/by-nc-nd/4.0/

\section{References}

1. Smith JM, Alloy LB. A roadmap to rumination: a review of the definition, assessment, and conceptualization of this multifaceted construct. Clin Psychol Rev 2009;29:116-28.

2. Watkins ER. Constructive and unconstructive repetitive thought. Psychol Bull 2008;134:163-206.

3. Nolen-Hoeksema S, Wisco BE, Lyubomirsky S. Rethinking rumination. Perspect Psychol Sci 2008;3:400-24.

4. Holdaway AS, Luebbe AM, Becker SP. Rumination in relation to suicide risk, ideation, and attempts: exacerbation by poor sleep quality? J Affect Disord 2018;236:6-13

5. Michalak J, Hölz A, Teismann T. Rumination as a predictor of relapse in mindfulness-based cognitive therapy for depression. Psychol Psychother 2011;84:230-6.

6. Spinhoven P, van Hemert AM, Penninx BW. Repetitive negative thinking as a predictor of depression and anxiety: a longitudinal cohort study. J Affect Disord 2018;241:216-25.

7. Spasojevic J, Alloy LB. Rumination as a common mechanism relating depressive risk factors to depression. Emotion 2001;1:25-37.

8. Abela JRZ, Hankin BL. Rumination as a vulnerability factor to depression during the transition from early to middle adolescence: a multiwave longitudinal study. J Abnorm Psychol 2011;120:259-71.

9. Nejad AB, Fossati P, Lemogne C. Self-referential processing, rumination, and cortical midline structures in major depression. Front Hum Neurosci 2013;7:666.

10. Qin P, Northoff G. How is our self-related to midline regions and the default-mode network? Neuroimage 2011;57:1221-33.

11. Berman MG, Peltier S, Nee DE, et al. Depression, rumination and the default network. Soc Cogn Affect Neurosci 2011;6:548-55.
12. Zamoscik V, Huffziger S, Ebner-Priemer U, et al. Increased involvement of the parahippocampal gyri in a sad mood predicts future depressive symptoms. Soc Cogn Affect Neurosci 2014;9:2034-40.

13. Zhu X, Wang X, Xiao J, et al. Evidence of a dissociation pattern in resting-state default mode network connectivity in first-episode, treatment-naive major depression patients. Biol Psychiatry 2012; 71:611-7.

14. Kaiser RH, Whitfield-Gabrieli S, Dillon DG, et al. Dynamic restingstate functional connectivity in major depression. Neuropsychopharmacology 2016;41:1822-30.

15. Philippi CL, Cornejo MD, Frost CP, et al. Neural and behavioral correlates of negative self-focused thought associated with depression. Hum Brain Mapp 2018;39:2246-57.

16. Grimm S, Boesiger P, Beck J, et al. Altered negative BOLD responses in the default-mode network during emotion processing in depressed subjects. Neuropsychopharmacology 2009;34:932-43.

17. Lemogne C, Mayberg H, Bergouignan L, et al. Self-referential processing and the prefrontal cortex over the course of depression: a pilot study. J Affect Disord 2010;124:196-201.

18. Yoshimura S, Okamoto Y, Onoda K, et al. Rostral anterior cingulate cortex activity mediates the relationship between the depressive symptoms and the medial prefrontal cortex activity. $J$ Affect Disord 2010;122:76-85.

19. Webb CA, Auerbach RP, Bondy E, et al. Reward-related neural predictors and mechanisms of symptom change in cognitive behavioral therapy for depressed adolescent girls. Biol Psychiatry Cogn Neurosci Neuroimaging 2021;6:39-49.

20. Auerbach RP, Bondy E, Stanton $\mathrm{CH}$, et al. Self-referential processing in adolescents: stability of behavioral and ERP markers. Psychophysiology 2016;53:1398-406.

21. Auerbach RP, Stanton $\mathrm{CH}$, Proudfit GH, et al. Self-referential processing in depressed adolescents: a high-density event-related potential study. J Abnorm Psychol 2015;124:233-45.

22. Shestyuk AY, Deldin PJ. Automatic and strategic representation of the self in major depression: trait and state abnormalities. Am J Psychiatry 2010;167:536-44.

23. Disner SG, Beevers CG, Haigh EAP, et al. Neural mechanisms of the cognitive model of depression. Nat Rev Neurosci 2011;12:467-77.

24. Gotlib IH, Kasch KL, Traill S, et al. Coherence and specificity of information-processing biases in depression and social phobia. J Abnorm Psychol 2004;113:386-98.

25. Mennen AC, Norman KA, Turk-Browne NB. Attentional bias in depression: understanding mechanisms to improve training and treatment. Curr Opin Psychol 2019;29:266-73.

26. Miskowiak KW, Larsen JE, Harmer CJ, et al. Is negative self-referent bias an endophenotype for depression? An fMRI study of emotional self-referent words in twins at high vs. low risk of depression. J Affect Disord 2018;226:267-73.

27. Johnson SC, Schmitz TW, Kawahara-Baccus TN, et al. The cerebral response during subjective choice with and without self-reference. J Cogn Neurosci 2005;17:1897-906.

28. Nakao T, Bai $Y$, Nashiwa H, et al. Resting-state EEG power predicts conflict-related brain activity in internally guided but not in externally guided decision-making. Neuroimage 2013;66:9-21.

29. Nakao T, Matsumoto T, Morita M, et al. The degree of early life stress predicts decreased medial prefrontal activations and the shift from internally to externally guided decision making: an exploratory NIRS study during resting state and self-oriented task. Front Hum Neurosci 2013;7:339.

30. Treynor W, Gonzalez R, Nolen-Hoeksema S. Rumination reconsidered: a psychometric analysis. Cognit Ther Res 2003;27:247-59.

31. Webb CA, Auerbach RP, Bondy E, et al. Abnormal neural responses to feedback in depressed adolescents. J Abnorm Psychol 2017;126:19-31.

32. Knyazev G. EEG correlates of self-referential processing. Front Hum Neurosci 2013;7:264.

33. Sheehan DV, Lecrubier Y, Sheehan KH, et al. The Mini-International Neuropsychiatric Interview (M.I.N.I.): the development and validation of a structured diagnostic psychiatric interview for DSM-IV and ICD-10. J Clin Psychiatry 1998;59(Suppl 20):22-33, quiz 34-57.

34. Beck AT, Steer RA, Ball R, et al. Comparison of Beck Depression Inventories -IA and -II in psychiatric outpatients. J Pers Assess 1996; 67:588-97.

35. Huang LJ, Wu CY, Wu CH, et al. Validation of the Ruminative Response Scale-Chinese Version (RRS-C) for persons with depression in Taiwan. Taiwan J Psychiatry 2015;29:119-31. 
36. Lu M. Reliability and validity of the Chinese version of the Beck Depression Inventory-II. Taiwan J Psychiatry 2002;16:301-10.

37. Buyukdura JS, McClintock SM, Croarkin PE. Psychomotor retardation in depression: biological underpinnings, measurement, and treatment. Prog Neuropsychopharmacol Biol Psychiatry 2011; 35:395-409.

38. Brainard DH. The psychophysics toolbox. Spat Vis 1997;10: 433-6.

39. Delorme A, Makeig S. EEGLAB: an open source toolbox for analysis of single-trial EEG dynamics including independent component analysis. J Neurosci Methods 2004;134:9-21.

40. Lopez-Calderon J, Luck SJ. ERPLAB: an open-source toolbox for the analysis of event-related potentials. Front Hum Neurosci 2014;8:213.

41. Herbert C, Pauli P, Herbert BM. Self-reference modulates the processing of emotional stimuli in the absence of explicit self-referential appraisal instructions. Soc Cogn Affect Neurosci Oxford Academic 2011;6:653-61.

42. JASP. Version 0.13.0. JASP Team; 2020. Available: https://jasp-stats. org/ (accessed 2021 Aug. 26).

43. Mensen A, Khatami R. Advanced EEG analysis using thresholdfree cluster-enhancement and non-parametric statistics. Neuroimage 2013;67:111-8.

44. Donoghue T, Haller M, Peterson EJ, et al. Parameterizing neural power spectra into periodic and aperiodic components. Nat Neurosci 2020;23:1655-65.

45. Ehinger B. behinger/eegvis: initial release (0.1). Geneva: Zenodo; 2018. Available: https://doi.org/10.5281/zenodo.1312813 (accessed 2021 Aug. 3).

46. Northoff G, Hirjak D, Wolf RC, et al. All roads lead to the motor cortex: psychomotor mechanisms and their biochemical modulation in psychiatric disorders. Mol Psychiatry 2021;26:92-102.

47. Kuiper NA, Derry PA. Depressed and nondepressed content selfreference in mild depressives. J Pers 1982;50:67-80.
48. Benau EM, Hill KE, Atchley RA, et al. Increased neural sensitivity to self-relevant stimuli in major depressive disorder. Psychophysiology 2019;56:e13345.

49. Hamilton JP, Farmer M, Fogelman P, et al. Depressive rumination, the default-mode network, and the dark matter of clinical neuroscience. Biol Psychiatry 2015;78:224-30.

50. Sheline YI, Barch DM, Price JL, et al. The default mode network and self-referential processes in depression. Proc Natl Acad Sci U S A 2009;106:1942-7.

51. Qin P, Wang M, Northoff G. Linking bodily, environmental and mental states in the self-a three-level model based on a metaanalysis. Neurosci Biobehav Rev 2020;115:77-95.

52. Allen JJB, Reznik SJ. Frontal EEG Asymmetry as a promising marker of depression vulnerability: summary and methodological considerations. Curr Opin Psychol 2015;4:93-7.

53. Suo $\mathrm{T}$, Liu $\mathrm{L}, \mathrm{Chen} \mathrm{C}$, et al. The functional role of individual-alpha based frontal asymmetry in the evaluation of emotional pictures: evidence from event-related potentials. Front Psychiatry 2017;8:180.

54. Young KD, Friedman ES, Collier A, et al. Response to SSRI intervention and amygdala activity during self-referential processing in major depressive disorder. Neuroimage Clin 2020;28:102388.

55. Fast LA, Funder DC. Gender differences in the correlates of selfreferent word use: authority, entitlement, and depressive symptoms. J Pers 2010;78:313-38

56. Mason NL, Kuypers KPC, Müller F, et al. Me, myself, bye: regional alterations in glutamate and the experience of ego dissolution with psilocybin. Neuropsychopharmacology 2020;45:2003-11.

57. Herbert C, Hesse K, Wildgruber D. Emotion and self in psychotic disorders: behavioral evidence from an emotional evaluation task using verbal stimuli varying in emotional valence and self-reference. J Behav Ther Exp Psychiatry 2018;58:86-96.

58. Ruscio AM, Gentes EL, Jones JD, et al. Rumination predicts heightened responding to stressful life events in major depressive disorder and generalized anxiety disorder. J Abnorm Psychol 2015;124:17-26. 